\title{
Action shots of the nuclear pore complex
}

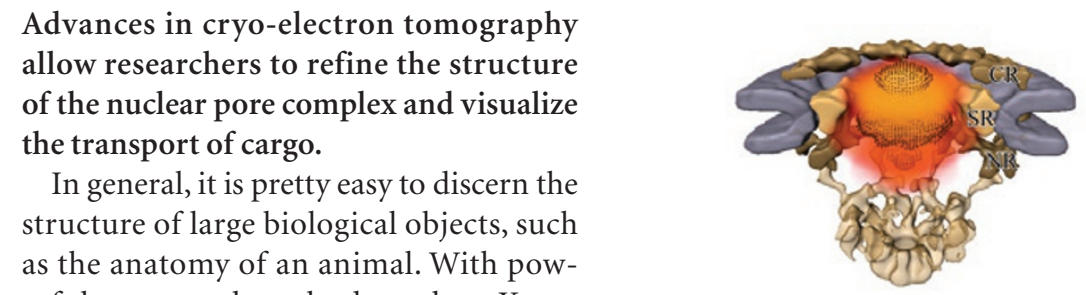

erful structural methods such as X-ray crystallography and NMR spectroscopy, it is also fairly straightforward to determine structures for very small objects such as individual proteins. But many interesting biological assemblies, such as large subcellular protein complexes, fall somewhere in between, and it is not so simple to analyze these structures.

Wolfgang Baumeister and colleagues at the Max Planck Institute for Biochemistry in Martinsried, Germany have long been involved in developing methodologies for cryo-electron tomography, a powerful tool ideally suited for looking at the structure of large subcellular assemblies, often in situ.

'Cryo' - you guessed it - means cold. In all cryo-electron microscopy techniques, biological samples are embedded in a layer of vitreous ice and imaged at extremely cold temperatures. The benefit of this, however, is that the close-to-life state of the cell is preserved. In single-particle cryo-electron microscopy, probably the best-known incarnation of cryo-electron microscopy, thousands of identical particles of macromolecular complexes are embedded in random orientations in the ice. A single image is then taken. With specialized imageprocessing methods, researchers determine the orientations of the individual particles with respect to each other and use this information to build a three-dimensional model of the structure.

Cryo-electron tomography differs in that a unique structure such as a cell or organelle is imaged multiple times at different tilt angles to collect enough information to build a three-dimensional model.
Figure 1 | Cryo-electron tomograms of the nuclear pore complex (cutaway image) showing the transport of gold-labeled cargo (orange cloud) through the central pore. CR, cytoplasmic ring; $S R$, spoke ring; NR, nuclear ring. Reprinted from Nature.

"No two cells are identical to each other," explains Baumeister, "so you must collect the [three-dimensional] information by tilting the specimen around an axis, and as you progress from one tilt angle to the next, you always take a new image."

As a test case for the methodological development of cryo-electron tomography, Baumeister and his colleagues have been working on the nuclear pore complex (NPC). Serving as a gatekeeper between the cytoplasm and nucleus, the NPC has the responsibility of controlling the transport of molecular cargo across the nuclear envelope. Made up of proteins called nucleoporins, the NPC weighs in at about 120 million daltons. The structure has eightfold symmetry and contains a central channel to allow cargo transport.

In 2004, Baumeister, graduate student Martin Beck and colleagues used cryoelectron tomography to obtain an 8.3-nm resolution structure of the NPC in intact nuclei from the organism Dictyostelium discoideum (Beck et al., 2004). Now, by developing a new image-processing strategy to deal with distortions from the perfect eightfold symmetry, Baumeister, Beck and their colleagues increased the resolution to $5.8 \mathrm{~nm}$ (Beck et al., 2007). This impressive achievement permitted the researchers to reveal several previously 'fuzzy' structural features.

But Baumeister had long been dreaming of expanding the capability of cryoelectron tomography to take 'snapshots' of cargo as it moved through the NPC. With the help of Ohad Medalia, an expert in gold nanoparticle labeling (which renders individual molecules visible in cryo-electron microscopy), the researchers generated a gold-labeled cargo: a model fusion protein made up of two copies of GFP and a nuclear localization signal. "We wanted to show that even though tomography is a static method, that by the superpositioning of many hundreds of tomograms of molecular structure you sort of take snapshots of different situations, in this case the cargo as it goes through the nuclear pore complex," explains Baumeister.

Notably, this work allowed the researchers to resolve that the observed electron density inside the channel corresponded to the goldlabeled cargo in transit "as some have speculated, but it was highly controversial," says Baumeister (Fig. 1). Previously, it was unclear whether this density represented cargo or a central plug in the pore.

It will certainly be exciting to see how cryoelectron tomography will be used to look at other subcellular structures, perhaps resolving other mechanistic debates. As for the nuclear pore complex, "We are determined to go one step further," says Baumeister. "From the current resolution, which is still relatively modest, I think it is entirely feasible to improve resolution by a factor of two; ... [this] will make a huge difference in detail."

\section{Allison Doerr}

\section{RESEARCH PAPERS}

Beck, M. et al. Snapshots of nuclear pore complexes in action captured by cryo-electron tomography. Nature 449, 611-615 (2007).

Beck, M. et al. Nuclear pore complex structure and dynamics revealed by cryoelectron tomography. Science 306, 1387-1390 (2004). 